\title{
Interaction of Ultraintense Laser Vortices with Plasma Mirrors
}

\author{
A. Denoeud, L. Chopineau, A. Leblanc, and F. Quéré* \\ LIDYL, CEA, CNRS, Université Paris-Saclay, CEA Saclay, 91191 Gif-sur-Yvette, France
}

(Received 1 September 2016; published 18 January 2017)

\begin{abstract}
Laser beams carrying orbital angular momentum (OAM) have found major applications in a variety of scientific fields, and their potential for ultrahigh-intensity laser-matter interactions has since recently been considered theoretically. We present an experiment where such beams interact with plasma mirrors up to laser intensities such that the motion of electrons in the laser field is relativistic. By measuring the spatial intensity and phase profiles of the high-order harmonics generated in the reflected beam, we obtain evidence for the helical wavefronts of the high-intensity laser at focus, and study the conservation of OAM in highly nonlinear optical processes at extreme laser intensities. The physical effects determining the field mode content of the twisted harmonic beams are elucidated.
\end{abstract}

DOI: 10.1103/PhysRevLett.118.033902

Light beams can carry both spin and orbital angular momenta in their direction of propagation. Their spin angular momentum is associated with the polarization state, whereas their orbital angular momentum (OAM) is related to the spatial structure of the wavefronts. Indeed, a beam with a helical spatial phase $\phi(r, \theta)=l \theta$, where $\theta$ is the azimuthal angle, carries an OAM of $l \hbar$ per photon, as is the case for the Laguerre-Gaussian (LG) eigenmodes of the paraxial wave equation of azimuthal index (or topological charge) $|l| \geq 1[1,2]$. Because of the phase singularity at $r=0$, the light intensity of such beams vanishes on the axis. These remarkable properties of so-called "twisted light beams" have led to applications going from optical manipulation [3-5] to high-resolution microscopy [6] or optical communication [7], based on laser beams typically in the infrared to visible spectral range.

In the last few years, there has been a growing interest for the use of such vortex laser beams to drive ultrahighintensity (UHI) laser-plasma interactions [8-16] up to the relativistic regime. These investigations are motivated by two main physical ideas. The first one is that the peculiar intensity profile of these beams provides new ways to control UHI interactions, e.g., leading to doughnut-shaped laser wakefields in underdense plasmas, suitable for efficient positron acceleration [8-10]. Secondly, the efficient transfer of OAM from optical vortices to plasmas could dramatically modify the physics of UHI interactions, thus leading to new effects of fundamental interest and new properties for the resulting light and particle beams [11-14].

Despite this recent surge of theoretical studies, there has been only one single UHI experiment carried out with such vortex laser beams, until now, where the hollow laser intensity profile was observed to lead to a reduction of the divergence of ion beams accelerated from thin solid foils [17]. This effect has been attributed to the doughnut shape of the laser focus, while the helicity of the laser wavefront and the associated OAM did not play a key role. This lack of experiments is partly due to the difficulty of inducing helical wavefronts on these large and powerful beams. To address this issue, different approaches, based on advanced laser-plasma interaction schemes, have been proposed theoretically or even demonstrated experimentally, such as plasma mirrors created on preformed helical targets [15], Raman amplification in underdense plasmas [16], or transient plasma holograms [18].

In this Letter, we present an experiment where highorder harmonics of the laser frequency are generated through the interaction of plasma mirrors $[19,20]$ with UHI laser vortices, produced by simply inserting a spiral phase plate in a high-power femtosecond laser beam just before focusing. By studying the amplitude and phase properties of these extreme ultraviolet (XUV) beams, we obtain clear evidence for the helical wavefronts of the laser beam on target at full power. From a fundamental point of view, we verify in an unambiguous way that the topological charge of the $n$th harmonic beam is $n$ times that of the driving laser beam, and thus, provide the first experimental validation [14] of the OAM conservation rule at such ultrahigh intensities. Furthermore, we analyze the key physical effects that determine the mode content of these beams, using a simple yet general approach that applies to any harmonic generation process driven by LG laser beams. This is, to the best of our knowledge, the first experiment demonstrating the controlled exchange of OAM between laser light and matter at relativistic intensities, opening the way of the exploitation of LG beams in UHI physics.

The experiment (see Supplemental Material in [21]) has been performed on UHI100, the $100 \mathrm{TW}$-class Ti:sapphire laser facility at CEA-IRAMIS. This laser delivers $25 \mathrm{fs}$ pulses at central wavelength $\lambda=800 \mathrm{~nm}$, with a contrast higher than $10^{12}$, obtained with a double plasma mirror [24]. The maximum energy on target is $0.6 \mathrm{~J}$, distributed in a 65 -mm-diameter beam. The scale length $L$ of the density gradient at the plasma-vacuum interface [25-27] was 
controlled by introducing a prepulse (fluence $\approx 10^{3} \mathrm{~J} / \mathrm{cm}^{2}$ ) that ionizes the optically flat silica target at an adjustable delay (between 0 and $5 \mathrm{ps}$ ) before the main pulse [25]. Its value was measured using spatial domain interferometry [26]. By controlling $L$, as well as the laser peak intensity $I$ (by adjusting the laser energy or the size of the main beam with an aperture mask), we could favor one or the other of the two identified high-order harmonic generation (HHG) mechanisms for plasma mirrors [25], namely coherent wake emission (CWE) or the relativistic oscillating mirror (ROM). Different optical components could be inserted in the path of the collimated beam before focusing by the $f=300 \mathrm{~mm}$ off-axis parabola. The laser beam was $p$ polarized and incident at $55^{\circ}$ on target.

We created the UHI optical vortex by inserting a $80-\mathrm{mm}-$ diameter 1-mm-thick silica spiral phase plate in the collimated beam. This 8-stair-step phase plate was designed to induce a helical phase with a $2 \pi$ variation per azimuthal turn $(l=1)$ at the central laser frequency. Figure $1 \mathrm{com}-$ pares the focal spots obtained with and without this spiral phase plate, measured at low laser power for two diameters of the apertured beam, $\phi=25 \mathrm{~mm}$ and $\phi=45 \mathrm{~mm}$. As expected, inserting the spiral phase plate leads to doughnutshaped focal spots. These spots, however, do not present a perfect azimuthal symmetry, but rather a lobe structure due to intensity inhomogeneities and residual phase aberrations in the laser beam [21].

At full power, the nonlinear phase, accumulated by the laser beam through this plate ( $B$ integral), is estimated to $1 \mathrm{rad}$, which is not expected to severely degrade the beam spatial properties [21]. The annular focal spots of Figs. 1(b) and 1(f) should thus, respectively, lead to peak intensities of a few $10^{17}$ and $10^{19} \mathrm{~W} / \mathrm{cm}^{2}$, high enough to drive HHG from plasma mirrors in the CWE and ROM regimes, respectively. We now confirm that such ultrahigh-intensity optical vortices are obtained on target by measuring the properties of the generated harmonic beams, using an angularly resolved XUV spectrometer.

Spectrally resolved 1D angular profiles of the harmonic beams, produced with the laser beam diaphragmed down to $\phi=25 \mathrm{~mm}$ and $\phi=45 \mathrm{~mm}$, are shown in Figs. 1(c) and $1(\mathrm{~g})$. These angular lineouts suggest that doughnut-shaped harmonic beams have been generated. To confirm this point, we rotated the solid target around its vertical axis by a small angle ( $\pm 45 \mathrm{mrad})$, thus sweeping the entire harmonic beam across the spectrometer entrance slit without affecting the generation conditions. By concatenating the resulting collection of angular lineouts, we could then recontruct the entire angular profile of each harmonic beam [21]. The results of this procedure are presented in Figs. 1(d) and 1(h) for the 12th harmonic, respectively, in the CWE and ROM regimes. In both cases, the harmonic beam presents a doughnut shape, although with an imperfect azimuthal symmetry, possibly reminiscent of that observed on the laser beam focus. These results clearly

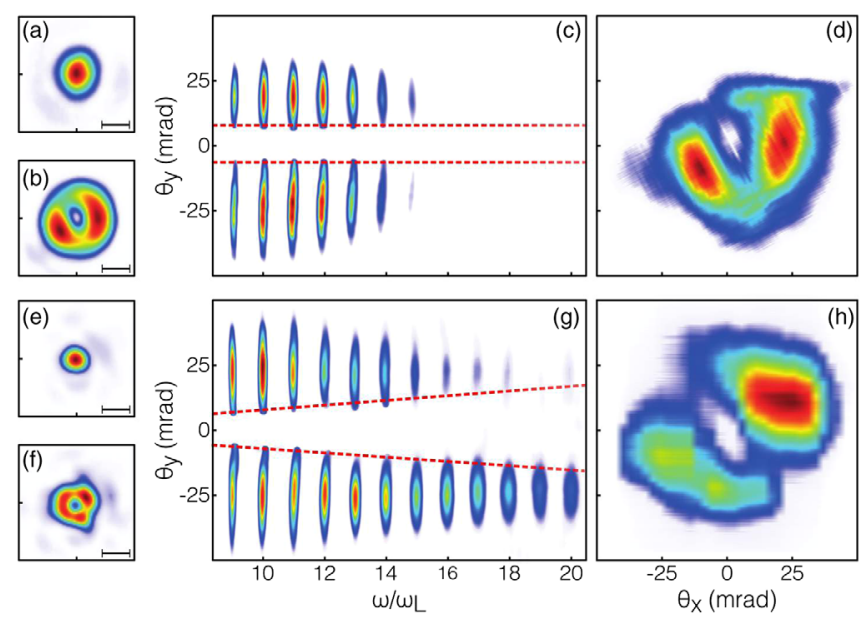

FIG. 1. Left panels: laser focal spots measured without (a),(e) and with (b),(f) the spiral phase plate in the path of a $\phi-25 \mathrm{~mm}$ (up) and $\phi-45 \mathrm{~mm}$ (down) diameter laser beam. The bar scale indicates a length of $10 \mu \mathrm{m}$. Central panels: raw images of the spectrally resolved high-order harmonic 1D angular profiles at $\theta_{x}=0 \mathrm{mrad}$, produced by the UHI vortices in the CWE (panel c, $L \approx \lambda / 70$ and $I \approx 5.10^{17} \mathrm{~W} / \mathrm{cm}^{2}$ ), and $\mathrm{ROM}$ (panel $\mathrm{g}, L \approx \lambda / 15$ and $I \approx 10^{19} \mathrm{~W} / \mathrm{cm}^{2}$ ) regimes. These values of $L$ were determined using the SDI interferometric technique described in [26]. Right panels: reconstructed 2D angular intensity profile of the 12th CWE (d) and ROM (h) harmonic beams.

indicate that the laser beam focus on target indeed has helical phase fronts at full power and that part of its OAM has been transferred to the XUV beams. We now focus on the physics of this transfer mediated by the plasma mirror.

Nonlinear processes driven by optical vortices have been the subject of multiple investigations in the last decades. In harmonic generation, the topological charge $l_{n}$ of the $n$th harmonic beam is expected to be $n$ times the one of the driving laser, $l_{n}=n l$, based on two physical arguments. First, as one harmonic photon is produced through the annihilation of $n$ laser photons, its OAM has to be $n$ times larger. Second, in any harmonic generation process, the phase of the $n$th harmonic is $n$ times that of the driving laser field: when the laser phase varies by $2 \pi l$ over one azimuthal turn, the harmonic phase should thus vary by $2 \pi l n$.

This conservation rule has been readily verified for loworder harmonics $[28,29]$. Its extension to high-order harmonics generated in gases at much higher intensities $\left(\approx 10^{14} \mathrm{~W} / \mathrm{cm}^{2}\right)$ has been somewhat controversial: while theoretical studies [30] and the latest experiments [31,32] confirmed this conservation rule, the first experiment in this regime rather suggested that $l_{n}=l[33]$-an unexpected result that has been attributed to the effect of propagation through the extended generation medium. Such potential complications are totally avoided in the present experiment, as the interaction occurs at an extremely steep plasmavacuum interface. Plasma mirrors are thus ideal to study the conservation of OAM in HHG at extreme laser intensities. 
To this end, we now need to experimentally access the spatial phase profile of the generated harmonic beams.

A standard way of determining the phase profile of a beam consists of measuring its interference pattern with a reference beam, e.g., one with spherical wavefronts. In the case of HHG, such a reference beam can be obtained by using a standard Gaussian laser focus for the generation [31]. We implement this scheme by placing a fork-shaped binary transmission grating in the collimated beam [21]—a method inspired from [34]. Such a grating is known to produce diffracted beams with a topological charge of $f k$ in the $k$ th order, where $f$ is the number of prongs of the fork ( $f=1$ here). This grating was designed so as to produce a central focal spot surrounded by two weaker satellites at a distance $a=16 \mu \mathrm{m}$, while higher-order satellites remained negligible. The resulting focal spot for a beam apertured to $43 \mathrm{~mm}$ is displayed in the inset of Fig. 2(a). As expected, it consists in a central Gaussian-like focal spot, surrounded by two optical vortices of topological charges 1 and -1 .

In these generation conditions, i.e., with significant energy losses induced by the transmission grating [21], each of these spots generates a CWE harmonic beam. The two lateral spots produce harmonic vortex beams, whose spatial phase we want to measure, whereas, the central one generates the reference wave for this measurement. As they diverge away from the target, these beams overlap and interfere spatially [Fig. 2(a)]. The resulting 2D interference pattern, reconstructed by sliding the transmission grating across the laser beam, is displayed in Fig. 2(b) for the 9th harmonic. It corresponds to a fork with multiple prongs whose number indicates the topological charge of the harmonic beam [21]. We measure a difference of $9 \pm 2$ fringes between the top and bottom of the interference pattern, clearly indicating a topological charge $l_{n} \gg 1$.

A more quantitative analysis can be performed by using a standard numerical procedure [21] to extract from the interferogram the phase difference between the interfering sources. This provides the phase profile shown in Fig. 2(c) where a fast azimuthal variation of the phase is observed. Its unwrapped variation over a complete azimuthal turn is plotted in Fig. 2(d), for the 9th, 11th, and 13th orders. Apart from some local deviations, most likely due to the inaccuracy of the phase retrieval in the low-intensity parts of the doughnut beams, these curves reveal a linear trend, with a slope equal to the harmonic order, i.e., $l_{n}=n$. This is clear evidence that there has been a transfer of OAM from the laser field to the high-order harmonics, mediated by the interaction with the plasma mirror, with the expected conservation law $l_{n}=n l[14]$.

We finally provide an insight into the spatial properties of the generated XUV vortices. As highlighted by the red dashed lines in Figs. 1(c) and 1(g), a striking feature in our experimental results is that the size of the central hole of the CWE vortices hardly varies with harmonic order $n$, while it does vary significantly for ROM vortices. We present a
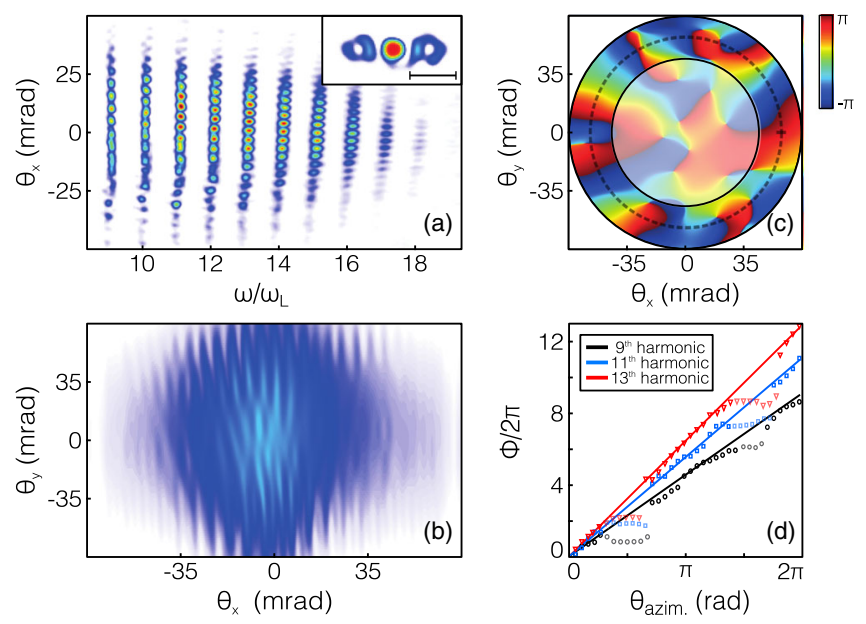

FIG. 2. (a) Experimental spectrally resolved interference pattern of the high-order harmonics, generated by the three focal spots presented in the inset (the bar scale corresponds to $20 \mu \mathrm{m}$ ). (b) Reconstructed 2D interference pattern of the 9th harmonic. The 2D phase profile retrieved from this interferogram is presented in (c), showing a variation of $9 \times 2 \pi$ of the phase over one azimuthal turn. The unwrapped azimuthal phases measured for the 9th, 11th, and 13th harmonics are presented as dots in (d) and compared with the theoretical expectation when OAM is conserved (full lines). All aspects of this measurement compare well with our numerical simulations detailed in [21].

simple analysis to understand these contrasted behaviors and relate these to the physics of the generation process. This analysis not only applies to HHG from plasma mirrors, but more generally to any harmonic generation process, e.g., HHG in gases.

We assume, for simplicity, that the driving laser field is a pure Laguerre-Gaussian mode, with an azimuthal index $l$ and a radial mode index $p=0\left(\mathrm{LG}_{p=0, l}\right)$ [Figs. 3(a) and 3(b)]. The spatial laser field on target is then given in cylindrical coordinates by

$$
E_{L}(r, \theta) \propto r^{l} \exp \left(-\frac{r^{2}}{w_{0}^{2}}+i l \theta\right) .
$$

For the sake of the analysis, let us first assume that the complex harmonic field $E_{n}$ is given by the $n$th power of the complex laser field, as is the case for harmonic generation in a perturbative regime. We then get

$$
E_{n}(r, \theta)=E_{L}^{n} \propto r^{n l} \exp \left(-\frac{r^{2}}{\left(w_{0} / \sqrt{n}\right)^{2}}+i n l \theta\right) .
$$

This expression exactly matches the definition of a pure Laguerre-Gaussian beam of indices $l_{n}=n l$ and $p=0$ $\left(\mathrm{LG}_{p=0, n l}\right)$. However, it is well known that HHG can generally not be described by such a perturbative behavior. When deviations from this power law occur, the harmonic beam is unavoidably produced in a superposition of 

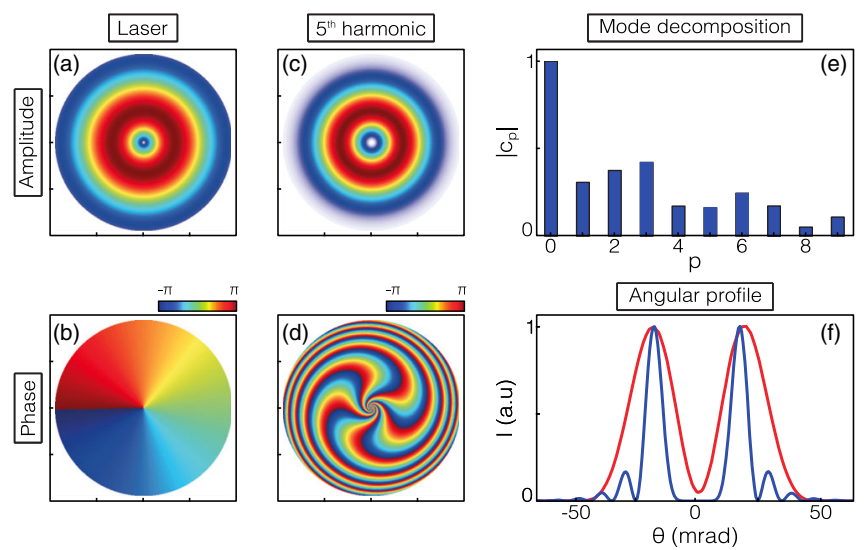

FIG. 3. Amplitude (a),(c) and phase (b),(d) profiles at focus of an ideal $\mathrm{LG}_{p=0, l=1}$ laser mode, and of the associated 5th harmonic beam calculated in a nonperturbative regime, using the CWE generation model [21]. (e) Absolute values $\left|c_{p}\right|$ of the coefficients of the multiple $\mathrm{LG}_{p, n l}$ modes contributing to the harmonic beam of panel (c),(d). (f) Lineouts of the resulting laser (red) and harmonic (blue) beam angular profiles.

multiple LG modes. Since the conservation law imposes a single value for the azimuthal index, $l_{n}=n l$, this mode superposition only involves the radial index $p$.

Two effects tend to broaden the $p$ distribution of the harmonic beam. The first one is related to the dependence of the harmonic amplitude on the laser amplitude: in HHG, this dependence $\left|E_{n}\right|=f\left(\left|E_{L}\right|\right)$ is typically weaker than $\left|E_{L}\right|^{n}$. As a result, the radial width of the harmonic ring $\left|E_{n}(r)\right|$ is larger than that of the pure $\mathrm{LG}_{p=0, n l}$ mode [Fig. 3(c)]. Second, in HHG, the harmonic phase $\phi_{n}$ has an intrinsic dependence on $\left|E_{L}\right|, \phi_{n}=\phi_{n}^{i}\left(\left|E_{L}(r)\right|\right)$. As $\left|E_{L}\right|$ varies with $r$, the total harmonic phase writes as $\phi_{n}(r, \theta)=n l \theta+$ $\phi_{n}^{i}\left(\left|E_{L}(r)\right|\right)$ [Fig. 3(d)]. These two deviations from the pure $\mathrm{LG}_{p=0, n l}$ mode can only be accounted for by superposing several LG modes with different $p$ [Fig. 3(e)], i.e., $E_{n}=\sum_{p} c_{p} \times \mathrm{LG}_{p, n l}$, where $c_{p}$ are complex coefficients.

As the harmonic beam propagates away from the source, the multiple constituting $\mathrm{LG}_{p, n l}$ modes experience different Gouy phase shifts $\psi(z)=(|n l|+2 p+1) \arctan \left(z / z_{R}\right)$. This modifies the relative phases of the modes in the superposition, and hence, affects the intensity profile measured at a distance from the source. This is illustrated in Fig. 3(f), in the case of the harmonic beam of Figs. 3(c) and 3(d). Instead of the simple initial doughnut in the focal plane, multiple rings are now observed due to the interference of LG modes with different $p$. In our experiment, these fine rings cannot be observed due to the insufficient spatial resolution of our detector, and they rather result in a broadening of the measured doughnut [Figs. 4(a) and 4(d)].

With these ideas in mind, we can now use a simple model of the laser-to-harmonic conversion process to explain the observed properties of the harmonic vortex beams [Figs. 1(c) and $1(\mathrm{~g})]$. To deduce the harmonic field at
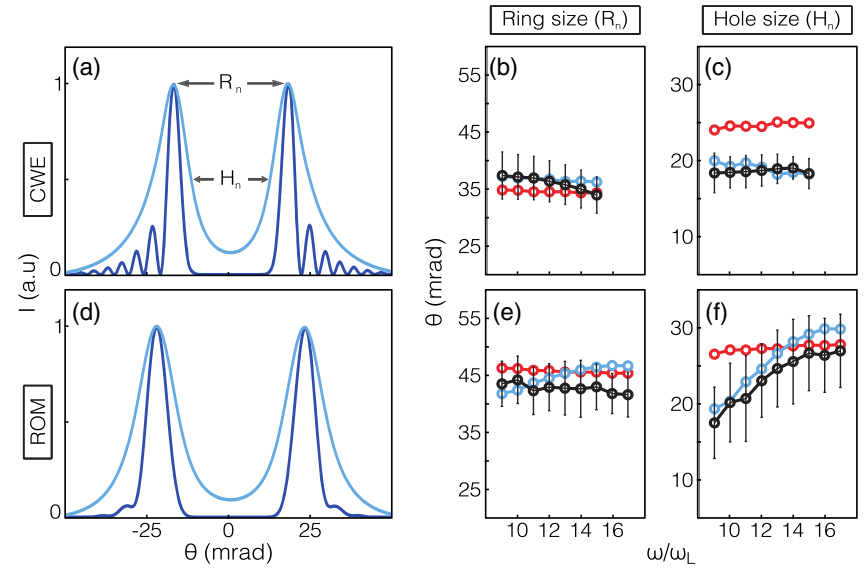

FIG. 4. Left panels: simulated angular intensity profiles of the 12th harmonic, with (light blue) and without (blue) convolution with the detector resolution ( $8 \mathrm{mrad}$ angular width). Right panels: comparison of the measured (black) and calculated (light blue) ring and hole diameters characterizing the harmonic vortex beams. The error bars correspond to the standard deviation, deduced from a statistical study on multiple laser shots. The upper and lower lines correspond to the CWE and ROM regime, respectively. The red lines show the prediction in the perturbative regime.

focus from the laser field given by Eq. (1), this model uses the known dependence of $\left|E_{n}\right|$ and $\phi_{n}^{i}$ on the laser amplitude $\left|E_{L}\right|$, determined in previous studies for both CWE and ROM [35-39] and reminded in [21]. It then propagates this field up to a detector by a Fourier transform. The predictions of this model are shown on the left panels of Fig. 4 for the 12th harmonic. With the spatial resolution of the detector taken into account, we then compare these predictions to the experimental results, using two parameters to describe the harmonic angular profiles: the ring diameter $R_{n}$ and the hole diameter $H_{n}$ [Fig. 4(a)]. The theoretical and experimental values of $R_{n}$ and $H_{n}$ are plotted in the right panels of Fig. 4 as a function of the harmonic order $n$ in the CWE and ROM regimes, showing a good agreement, despite the simplicity of the model. In contrast, significant deviations are observed for the simple perturbative model.

Using this model for a detailed analysis of the HHG vortices properties shows that the ring diameter $R_{n}$ is mostly determined by the $l$ index. The measured values of $R_{n}$ can only be reproduced when $l_{n}=n l$. Indeed, using $l_{n}=l$, as suggested in [33], leads to values of $R_{n}$ about five times smaller than observed experimentally [21]. As far as the hole size $H_{n}$ is concerned, its evolution is mostly determined by the intensity dependence of harmonic phase for CWE and the intensity dependence of the harmonic amplitude for ROM [21]. This is what leads to the contrasted properties of the harmonic vortices in these two regimes.

In conclusion, we have demonstrated the feasibility of ultrahigh-intensity laser-plasma interaction experiments 
with LG beams and reported the first experimental observations where the helical phase of these beams comes into play at these laser intensities. An important challenge for future studies on such interaction will be to find ways to efficiently deposit the laser OAM into plasmas to induce new physical effects in UHI physics. Besides the fundamental aspects investigated here, this Letter establishes a new methodology for the generation of intense XUV vortices [40-43]. Such vortices, which form spatiotemporal light springs when multiple harmonic orders are superimposed [44], might find intriguing applications as advanced probes of matter.

We acknowledge the expert support from the UHI100 laser group and the mechanical workshop, as well as R. Géneaux and T. Ruchon for useful discussions. The research leading to these results has received funding from Agence Nationale pour la Recherche (Project No. ANR-14CE32-0011) and from the European Research Council under the European Union's Horizon 2020 research and innovation programme (ERC Grant No. 694596).

*fabien.quere@cea.fr

[1] L. Allen, M. W. Beijersbergen, R. J. C. Spreeuw, and J. P. Woerdman, Phys. Rev. A 45, 8185 (1992).

[2] A. M. Yao and M. J. Padgett, Adv. Opt. Photonics 3, 161 (2011).

[3] H. He, M. E. J. Friese, N. R. Heckenberg, and H. Rubinsztein-Dunlop, Phys. Rev. Lett. 75, 826 (1995).

[4] D. G. Grier, Nature (London) 424, 810 (2003).

[5] M. J. Padgett and R. Bowman, Nat. Photonics 5, 343 (2011).

[6] K. I. Willig, S. O. Rizzoli, V. Westphal, R. Jahn, and S. W. Hell, Nature (London) 440, 935 (2006).

[7] J. Wang et al., Nat. Photonics 6, 488 (2012).

[8] J. T. Mendonca and J. Vieira, Phys. Plasmas 21, 033107 (2014).

[9] J. Vieira and J. T. Mendonca, Phys. Rev. Lett. 112, 215001 (2014).

[10] G.-B. Zhang et al., J. Appl. Phys. 119, 103101 (2016).

[11] G.-B. Zhang et al., Phys. Plasmas 23, 033114 (2016).

[12] W. Wang, B. Shen, X. Zhang, L. Zhang, Y. Shi, and Z. Xu, Sci. Rep. 5, 8274 (2015).

[13] Z. Lécz, A. Andreev, and A. Seryi, Laser Part. Beams 34, 31 (2016).

[14] X. Zhang, B. Shen, Y. Shi, X. Wang, L. Zhang, W. Wang, J. Xu, L. Yi, and Z. Xu, Phys. Rev. Lett. 114, 173901 (2015).

[15] Y. Shi, B. Shen, L. Zhang, X. Zhang, W. Wang, and Z. Xu, Phys. Rev. Lett. 112, 235001 (2014).

[16] J. Vieira, R. M. G. M. Trines, E. P. Alves, R. A. Fonseca, J. T. Mendonca, R. Bingham, P. Norreys, and L. O. Silva, Nat. Commun. 7, 10371 (2015).

[17] C. Brabetz, S. Busold, T. Cowan, O. Deppert, D. Jahn, O. Kester, M. Roth, D. Schumacher, and V. Bagnoud, Phys. Plasmas 22, 013105 (2015).
[18] A. Leblanc, L. Chopineau, A. Denoeud, G. Mennerat, and F. Quéré (to be published).

[19] U. Teubner and P. Gibbon, Rev. Mod. Phys. 81, 445 (2009).

[20] C. Thaury and F. Quéré, J. Phys. B 43, 213001 (2010).

[21] See Supplemental Material at http://link.aps.org/ supplemental/10.1103/PhysRevLett.118.033902, which includes Refs [22,23], for details on the experimental setup and data analysis.

[22] B. Chimier, O. Utéza, N. Sanner, M. Sentis, T. Itina, P. Lassonde, F. Légaré, F. Vidal, and J. C. Kieffer, Phys. Rev. B 84, 094104 (2011).

[23] M. Takeda, H. Ina, and S. Kobayashi, J. Opt. Soc. Am. 72, 156 (1982).

[24] A. Lévy et al., Opt. Lett. 32, 310 (2007).

[25] S. Kahaly, S. Monchocé, H. Vincenti, T. Dzelzainis, B. Dromey, M. Zepf, Ph. Martin, and F. Quéré, Phys. Rev. Lett. 110, 175001 (2013).

[26] M. Bocoum, F. Böhle, A. Vernier, A. Jullien, J. Faure, and R. Lopez-Martens, Opt. Lett. 40, 3009 (2015).

[27] Y. B. Zeldovich and Y. P. Raizer, Physics of Shock Waves and High-Temperature Hydrodynamic Phenomena (Dover Publications, New York, 2002).

[28] K. Dholakia, N. B. Simpson, M. J. Padgett, and L. Allen, Phys. Rev. A 54, R3742(R) (1996).

[29] J. Courtial, K. Dholakia, L. Allen, and M. J. Padgett, Phys. Rev. A 56, 4193 (1997).

[30] C. Hernández-García, A. Picón, J. San Román, and L. Plaja, Phys. Rev. Lett. 111, 083602 (2013).

[31] G. Gariepy, J. Leach, K. T. Kim, T. J. Hammond, E. Frumker, R. W. Boyd, and P. B. Corkum, Phys. Rev. Lett. 113, 153901 (2014).

[32] R. Géneaux, A. Camper, T. Auguste, O. Gobert, J. Caillat, R. Taeib, and T. Ruchon, Nat. Commun. 7, 12583 (2015).

[33] M. Zürch, C. Kern, P. Hansinger, A. Dreischuh, and C. Spielmann, Nat. Phys. 8, 743 (2012).

[34] C. Thaury, H. George, F. Quéré, R. Loch, J.-P. Geindre, P. Monot, and Ph. Martin, Nat. Phys. 4, 631 (2008).

[35] F. Quéré, C. Thaury, J.-P. Geindre, G. Bonnaud, P. Monot, and Ph. Martin, Phys. Rev. Lett. 100, 095004 (2008).

[36] A. Malvache, A. Borot, F. Quéré, and R. Lopez-Martens, Phys. Rev. E 87, 035101 (2013).

[37] H. Vincenti, S. Monchocé, S. Kahaly, G. Bonnaud, Ph. Martin, and F. Quéré, Nat. Commun. 5, 3403 (2014).

[38] A. Leblanc, S. Monchocé, C. Bourassin-Bouchet, S. Kahaly, and F. Quéré, Nat. Phys. 12, 301 (2016).

[39] A. Leblanc, S. Monchocé, H. Vincenti, S. Kahaly, and F. Quéré (to be published).

[40] S. Sasaki and I. McNulty, Phys. Rev. Lett. 100, 124801 (2008).

[41] J. Bahrdt, K. Holldack, P. Kuske, R. Müller, M. Scheer, and P. Schmid, Phys. Rev. Lett. 111, 034801 (2013).

[42] P. R. Ribič, D. Gauthier, and G. De Ninno, Phys. Rev. Lett. 112, 203602 (2014).

[43] E. Hemsing, M. Dunning, C. Hast, T. Raubenheimer, and D. Xiang, Phys. Rev. Lett. 113, 134803 (2014).

[44] G. Pariente and F. Quéré, Opt. Lett. 40, 2037 (2015). 